\title{
Bioassay-guided identification of an anti-inflammatory prenylated acylphloroglucinol from Melicope ptelefolia and molecular insights into its interaction with 5-lipoxygenase.
}

\begin{abstract}
A bioassay-guided investigation of Melicope ptelefolia Champ ex Benth (Rutaceae) resulted in the identification of an acyphloroglucinol, 2,4,6-trihydroxy-3-geranylacetophenone or tHGA, as the active principle inhibiting soybean 15-LOX. The anti-inflammatory action was also demonstrated on human leukocytes, where the compound showed prominent inhibitory activity against human PBML 5-LOX, with an IC 50 value of $0.42 \mu \mathrm{M}$, very close to the effect produced by the commonly used standard, NDGA. The compound concentrationdependently inhibited 5-LOX product synthesis, specifically inhibiting cysteinyl leukotriene LTC4 with an IC 50 value of $1.80 \mu \mathrm{M}$, and showed no cell toxicity effects. The antiinflammatory action does not seem to proceed via redox or metal chelating mechanism since the compound tested negative for these bioactivities. Further tests on cyclooxygenases indicated that the compound acts via a dual LOX/COX inhibitory mechanism, with greater selectivity for 5-LOX and COX-2 (IC 50 value of $0.40 \mu \mathrm{M}$ ). The molecular features that govern the 5-LOX inhibitory activity was thus explored using in silico docking experiments. The residues Ile 553 and Hie 252 were the most important residues in the interaction, each contributing significant energy values of 13.45 (electrostatic) and $5.40 \mathrm{kcal} / \mathrm{mol}$ (electrostatic and Van der Waals), respectively. The hydroxyl group of the phloroglucinol core of the compound forms a $2.56 \AA$ hydrogen bond with the side chain of the carboxylate group of Ile 553. Both Ile 553 and Hie 252 are crucial amino acid residues which chelate with the metal ion in the active site. Distorting the geometry of these ligands could be the reason for the inhibition activity shown by tHGA. The molecular simulation studies supported the bioassay results and served as a good model for understanding the way tHGA binds in the active site of human 5-LOX enzyme.
\end{abstract}

Keyword: Melicope ptelefolia; Rutacecae; Acylpholoroglucinol; Lipoxygenase; Cycloxigenase,; Anti-inflammatory. 